\title{
AN 8-BIT DAC IMPLEMENTATION USING SINGLE ELECTRON TUNNELING (SET) TECHNOLOGY
}

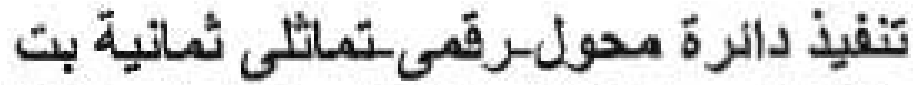

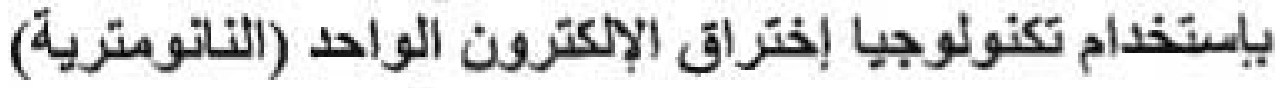

Dr. Sameh Ebrahim Reban and Eng. Lobna Abd Elaziz Osman

Communications and Electronics Engineering Department,

Faculty of Engineering, Mansoura University,

Mansoura, EGYPT 35516

Email: sameh rehan@ieee.org

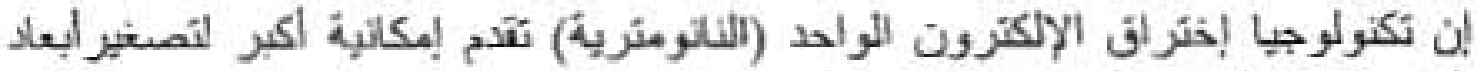

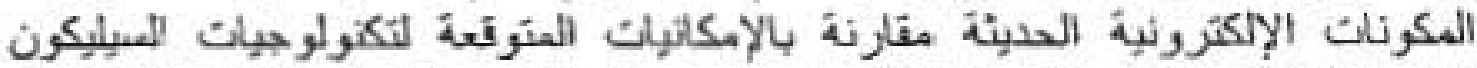

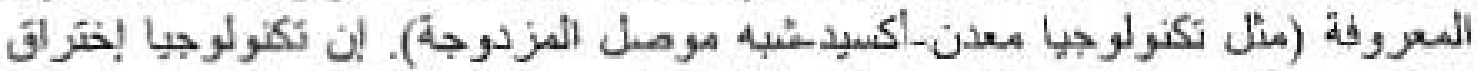

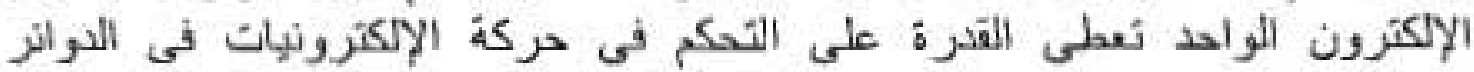

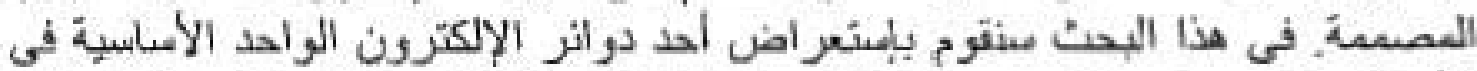

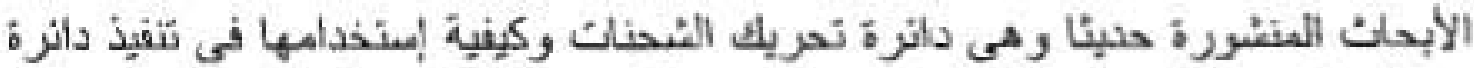

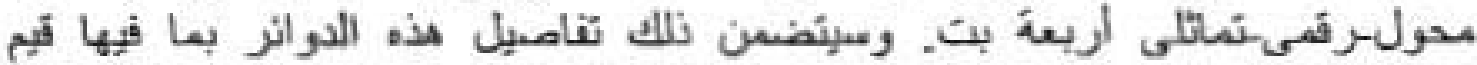

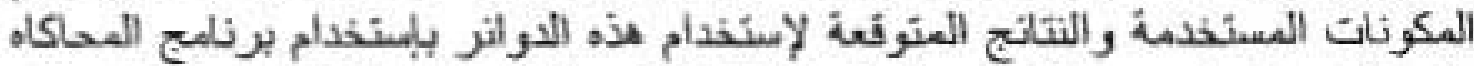

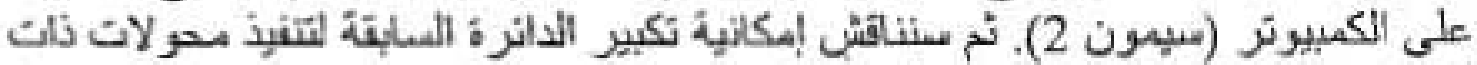

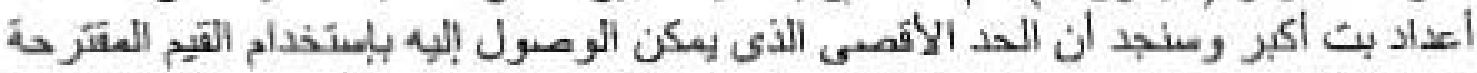

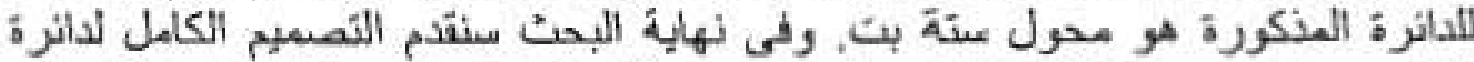

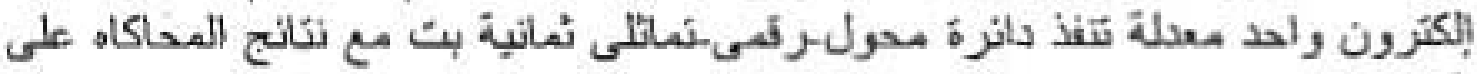
الكبيونر

Abstract:

An 8-bit Digital-to-Analog Converter (DAC) based on the Multiple-Value k electron (MVke) Single Eleetron Circuit (SEC) is presented. With each MVke SEC, a variable number of electrons $k^{*} e$ can be added one by one to an output node. Each MVke SEC, consists of two tunnel junctions and five capacitors. The proposed 8-bit DAC consists of eight MVke SEC blocks in addition to the charge reservoir load capacitor. It is implemented and simulated using SIMON 2.

Keywords:

Single Electron Tunneling (SET), Singie Electron Circuit (SEC), Digital to Analog Converter (DAC), Multiple-Value k electron (MVke). 


\section{E. 19 Sameh Ebrahim Rehan \& Lobna Abd.Elaziz Osman.}

\section{Introduction:}

The Single Electron Tunneling (SET) technology is the future technology that is expected to meet the required increase in density and performance and decrease in power dissipation [1-2]. The main device of the SET circuits is the tunnel junction through which individual electrons can move in a controlled manner [3].

A decade ago, the basic physics of SET was well understood and designing useful Single Electron Circuits (SECs) became the important research area [4. 5]. In the past few years, some basic building blocks for SEC had been introduced in the literature [6-10].

In this paper, we first briefly discuss the basic physics of SET in section 2 . In Section 3, we review a basic SEC building block that was introduced in the literature; the Multiple-Value $k$ electron (MVke) block [3] Based on the MVke SEC block, we present an 8-bit Digitalto-Analog Converter (DAC) implementation in Section 4. The full design of the 8-bit SEC DAC (inc. detailed schematic diagrams with all parameters for used devices) and simulation results (using the Monte Carlo simulator, SIMON 2 [I H] are included. The conclusions are provided in Section 5.

\section{The Basic Physics of SET:}

The main component of SEC is the tunnel junction that can be implemented using silicon or metal-insulator-metal structures, GaAs quantum dots, etc.

The tunnel junction can be thought of as a leaky capacitor [II]. For very small tunnel junctions (hence, very small capacitance $C_{1}$, , the movement of only one electron, from one side of the tunnel junction to the other, may produce a noticeable change $\mathrm{e} / \mathrm{C}$ of the voltage across the tunnel junction. Note that the above $\mathrm{C}=\mathrm{C}_{j}+\mathrm{C}_{\text {}}$ where $\mathrm{C}_{\mathrm{e}}$ is the equivalent capacitance of the remainder of the circuit, as viewed from the tunnel junction's perspective.

The discreteness of the electrical charge $c$ leads to the Coulomb blockade effect that is widely known in the field of single-electronics. The critical voltage $\mathrm{V}_{\epsilon}$ (the voltage needed in order to make one electron tunnel through the junction) is given by [6]:

$V_{e}=e / 2 C$

The Coulomb blockade effect is the suppression of electron tunneling across the tunael junction at voltages $|V|<$ $\mathrm{e} / 2 \mathrm{C}$. This means that for such voltages, there will be no increase in the electrostatic energy of the junction capacitor: $\mathrm{CV}^{2} / 2$ (in the case of such increase, the energy would be [4]: $\mathrm{C}(\mathrm{V} \pm \mathrm{e} / \mathrm{C})^{2} / 2$.

Today's well-established technologies uses metal junctions with an area about $50 \times 50 \mathrm{~nm}^{2}$ that lead to a typical capacitance and its corresponding voltage scale $(\mathrm{e} / \mathrm{C})$ in the order of $100 \mathrm{af}$ and I $\mathrm{mV}$, respectively.

To avoid the effects of thermal fluctuations on SEC, the thermal energy $\mathrm{k}_{\mathrm{o}} \mathrm{T}$ should be much less than the typical one-electron charging energy ( $\mathrm{eV}=\mathrm{e}^{2}$ /2C) [4],

$k_{\mathrm{B}} \mathrm{T} \ll \mathrm{e}^{2} / 2 \mathrm{C}$ 
The above condition limits the practical use of SEC since the working temp. is restricted to be $<1 \mathrm{~K}$. For a room operating temperature $(300 \mathrm{~K})$, junction capacitances should decrease to the range of $0.1 \mathrm{aF}$. To reach this low capacitance level, the size of the devices should go below few nanometers and single electronics enters the areas of atomic physics and chemistry [4].

\section{Review of the MVke SEC Block:}

The MVke SEC block [3], shown in Fig. 1 , is a SEC basic block with which a variable number of electrons ke can be added to the charge reservoir (CR) output node where $\mathrm{k}$ is determined by the input voltage $V$. The inputs: Enable $(E)$ and Reset (R) are control signals. The MVke block has a dynamic logic behavior, therefore it has to be reset before each new charge transport. This reset can be achieved by setting $R=$ " 1 " and $\mathrm{E}=$ " 0 ".

Considering $\mathrm{C}=\mathrm{I}$ aF as the unit of capacitance for the MVke block, logic "l" $=q^{*} \mathrm{e} / 10^{*} \mathrm{C}=16 \mathrm{mV}$ and logic " 0 " $=0 \mathrm{~V}$. Let $\mathrm{C}_{\Sigma i}$ and $\mathrm{C}_{\Sigma i}$ be the equivalent capacitances at internal nodes $\mathrm{t}$ and $\mathrm{i}$, respectively [3].

$$
\begin{aligned}
& C_{\Sigma t}=C_{j 1}+C_{e}+C_{v}+C_{t} \\
& C_{\Sigma i}=C_{\jmath 1}+C_{j 2}+C_{r}+C_{i}
\end{aligned}
$$

In [3], the following values were chosen and/or calculated for the various MVke elements:

$$
\begin{aligned}
& C_{j 1}=C_{j 2}=0.5 \mathrm{C}=0.5 \mathrm{aF} \\
& . C_{\Sigma i}=10 \mathrm{C}=10 \mathrm{aF} \\
& C_{r} / C_{\Sigma i}=5 / 10 \\
& C_{r}=5 \mathrm{aF} \\
& C_{i}=4 \mathrm{aF}
\end{aligned}
$$

$$
\begin{aligned}
& \mathrm{C}_{\mathrm{e}} / \mathrm{C}_{\Sigma \mathrm{t}}=5 / 9.5 \\
& \mathrm{C}_{\mathrm{cr}}=10000 \mathrm{aF}=10 \mathrm{fF}
\end{aligned}
$$

For $\mathrm{V}=1, \mathrm{k}=\mathrm{C}_{\mathrm{v}} / \alpha^{*} \mathrm{C}$, where $\alpha$ (amplification factor) $=100$. Therefore $\mathrm{k}$ is determined by $C_{v}$. For example, $C_{v}=$ $300 \mathrm{aF} \rightarrow \mathrm{k}=3$.

In [3], an MVke block was simulated (using SIMON [11]) with the following values: $\mathrm{C}_{\mathrm{e}}=500 \mathrm{aF}, \mathrm{C}_{\mathrm{v}}=300 \mathrm{aF}$, and $\mathrm{C}_{\mathrm{t}}$ $=150 \mathrm{aF}$. Notice that equation $(10)$ is still valid. It is worth noting here that:

1. Every electron transfer to the charge reservoir output node $\mathrm{CR}$ corresponds to an increase of $16 \mathrm{uV}$ in its voltage $\mathrm{V}_{\mathrm{cr}}$.

2. $V_{\mathrm{cr}}$ maintains its current value when $\mathrm{E}=\mathrm{R}=\mathrm{V}=0 \mathrm{~V}$.

3. $V_{\mathrm{cr}}$ maintains its previous value (no charge transport occurs) while $E=0$ $V$ (even if input $V$ changes).

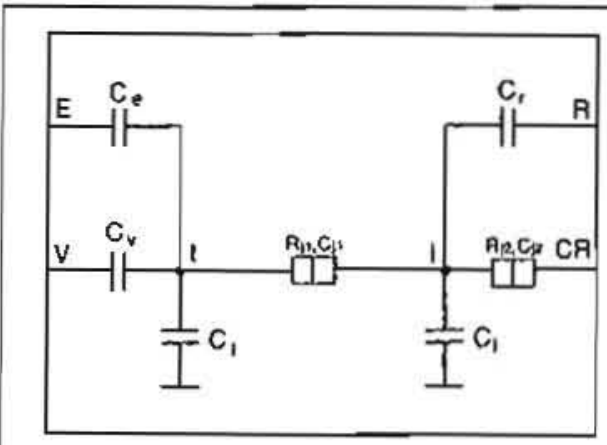

Fig. 1 Circuit Diagram of the MVke SEC block.

\section{The 8-Bit DAC SEC:}

\section{A. The reported 4-bit DAC SEC:}

A 4-bit DAC SEC was implemented using the MVke block. Four MVke blocks were used to implement the 4-bit DAC SEC. For each MVke block, $C_{v}$ had to be computed for the desired $k$ [3]. Alt other circuit parameters were kept as presented in Section 3 except for the $C_{t}$ 


\section{E. 21 Sameh Ebrahim Rehan \& Lobna Abd-Eluziz Osman.}

values. Keeping $C_{E}=500$ af, $C_{1}$ is calculated using the equation $C_{t}=450$ $\mathrm{aF}-\mathrm{C}_{\text {. }}$. The calculated values for $\mathrm{C}_{2}$ and $\mathrm{C}_{1}$ are listed in Table 1.

Table 1: The values of $C_{\text {, }}$ for the 4-bit DAC SEC

\begin{tabular}{|c|c|c|c|c|}
\hline $\begin{array}{c}\text { MVke } \\
\text { block }\end{array}$ & Inputs & $\mathrm{k}$ & $\begin{array}{c}\mathrm{C}_{\mathrm{v}} \\
(\mathrm{aF})\end{array}$ & $\begin{array}{c}\mathrm{C}_{1} \\
(\mathrm{a} F)\end{array}$ \\
\hline 1 & $\mathrm{D} 0$ & 1 & 10 & 440 \\
\hline 2 & $\mathrm{D} 1$ & 2 & 20 & 430 \\
\hline 3 & $\mathrm{D} 2$ & 4 & 40 & 410 \\
\hline 4 & D3 & 8 & 80 & 370 \\
\hline
\end{tabular}

\section{B. The developed 8-bit DAC SEC:}

Using the circuit parameters reported in [3]. we were able to extend the DAC design to 6-bit. The 6-bit DAC SEC can be implemented using the values listed in both Table I and Table 2.

Table 2: The extra values of $\mathrm{C}$, for the 6-bit DAC SEC

\begin{tabular}{|c|c|c|c|c|}
\hline $\begin{array}{c}\text { MVke } \\
\text { block }\end{array}$ & Inputs & $k$ & $\begin{array}{c}\mathrm{C}_{V} \\
(\mathrm{aF})\end{array}$ & $\begin{array}{c}\mathrm{C}_{\mathrm{t}} \\
(\mathrm{aF})\end{array}$ \\
\hline 5 & $\mathrm{D} 4$ & 16 & 160 & 290 \\
\hline 6 & $\mathrm{D} 5$ & 32 & 320 & 130 \\
\hline
\end{tabular}

Using the values proposed in [3], we can not extend the DAC design any further (because that value of $C_{v}$ will need to go beyond the available range of values).

In this paper, we propose the following changes to the original circuit parameters to be able to achieve 8-bit DAC SEC using the MVke SEC block developed in [3];
1. Increasing the charge reservoir load capacitance $C_{a}$ value by ten fold to $100 \mathrm{fF}$,

2- Increasing the $\mathrm{C}_{t}$ value by three folds to 1500 af and maintaining equation (7) so that we can achieve 8-bit DAC SEC. $C_{1}$ is calculated using the equation $\mathrm{C}_{1}=$ $1350 \mathrm{aF}-\mathrm{C}_{\mathrm{r}}$. The new values for $\mathrm{C}_{v}$ and $\mathrm{C}_{1}$ are listed in Table 3.

Table 3: The values of $\mathrm{C}_{v}$ for the 8-bit DAC SEC

\begin{tabular}{|c|c|c|c|c|}
\hline $\begin{array}{c}\text { MVke } \\
\text { block }\end{array}$ & Inputs & $k$ & $\begin{array}{c}\mathrm{C}_{2} \\
(\mathrm{aF})\end{array}$ & $\begin{array}{c}\mathrm{C}_{\mathrm{t}} \\
(\mathrm{aF})\end{array}$ \\
\hline 1 & D0 & 1 & 10 & 1340 \\
\hline 2 & D1 & 2 & 20 & 1330 \\
\hline 3 & D2 & 4 & 40 & 1310 \\
\hline 4 & D3 & 8 & 80 & 1270 \\
\hline 5 & D4 & 16 & 160 & 1190 \\
\hline 6 & D5 & 32 & 320 & 1030 \\
\hline 7 & D6 & 64 & 640 & 710 \\
\hline 8 & D7 & 128 & 1280 & 70 \\
\hline
\end{tabular}

The schematic diagram of the proposed 8-bit DAC SEC is shown in Fig. 2. The detailed 8-bit DAC SEC (using the circuit parameters listed in this paper) was implemented and simulated using SIMON 2 [11]. Various 8-bit numbers are considered as input to the proposed 8-bit DAC SEC.

Fig. 3 shows the simulation results of the proposed 8-bit DAC SEC for a selected series of 20 input samples using SIMON 2 [11]. Table 4 shows the charge 
reservoir output voltage $V_{c r}$ for the selected series of 8-bit inputs.

It should be noted here that $E$ must equal "I" so that digital-to-analog conversion is performed. Otherwise (i.e., $E=" 0$ "), the charge reservoir remains in the neutral state (i.e., the output voltage $\mathrm{V}_{\mathrm{ct}}$ equals " 0 ". This can be noticed by looking into the reported values in the rows representing the input samples 12 and 20 within Table 4.

Since we have 8-bit DAC, input can have 256 integer values starting from 0 to 255 . It can be noticed from Table 4 that for each increase of 1 in the input, the charge reservoir output voliage $\mathrm{V}_{\mathrm{ct}}$ increases by $1.6 \mu \mathrm{V}$. There will also be a decrease of $1.6 \mu \mathrm{V}$ in the charge reservoir output voltage $V_{c r}$ for each decrease of $I$ in the input.

Both Table 4 and Fig. 3 indicate that the proposed 8-bit DAC SEC performs the correct conversions for both increasing and decreasing inputs. This can be attributed to the introduction of the reset (R) signal $(\mathrm{R}=$ " $\mid$ ") before applying any new input sample.

It should be noted here that by reducing the charge reservoir capacitance value $\mathrm{C}_{\mathrm{cr}}$, the corresponding voltage $\mathrm{V}_{\mathrm{cr}}$ increases. For example, when $\mathrm{C}_{\mathrm{cr}}=4 \mathrm{fF}$ is used, a step increase of about $40 \mu \mathrm{V}$ is achieved (rather than the $1.6 \mu \mathrm{V}$ that was achieved when $\mathrm{C}_{\mathrm{cr}}=100 \mathrm{fF}$ is used. This has the effect of increasing the maximum charge reservoir output voltage $V_{c r}$ from about $400 \mu \mathrm{V}$ to about $8.4 \mathrm{mV}$ as can be seen in Fig. 4. This came at the expense of having smaller voltage steps for higher bit representations (i.e. the charge reservoir output voltage $\mathrm{V}_{\mathrm{cr}}$ tends to saturates when input increases). For example, instead of getting $10.2 \mathrm{mV}$ for the maximum input representation, we only get $8.4 \mathrm{mV}$.

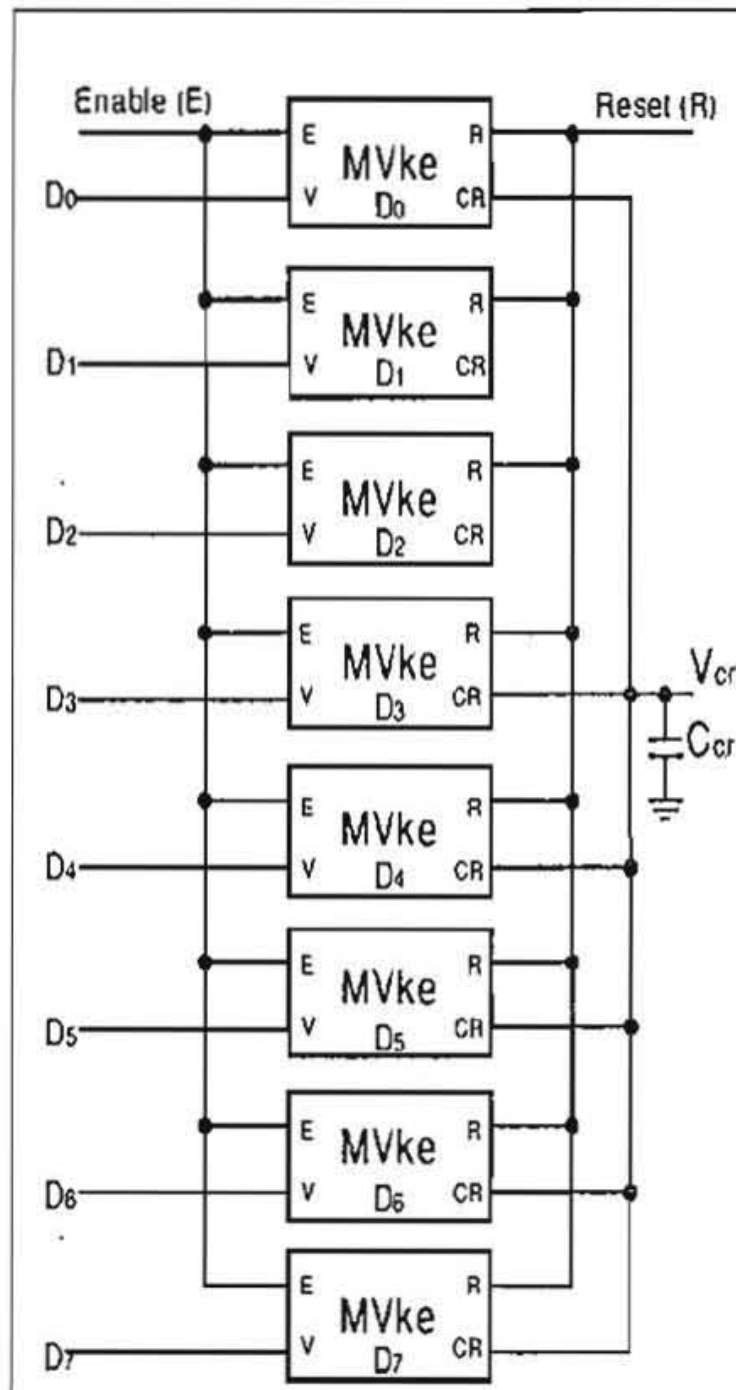

Fig. 2 Schematic Diagram of the 8-bit DAC.

\section{Conclusions}

In this paper we reviewed the MVke SEC block and the design of a 4-bit DAC that was introduced in [3] using this block. It was shown that using the circuit parameters suggested in the above reference, the DAC design can be expanded to only 6-bit. We introduced some modifications to circuit parameters in order to be able to achieve 8-bit DAC SEC. 
E. 23 Sameh Ebruhim Rchan \& Lobna Abd-Elaziz Osman.

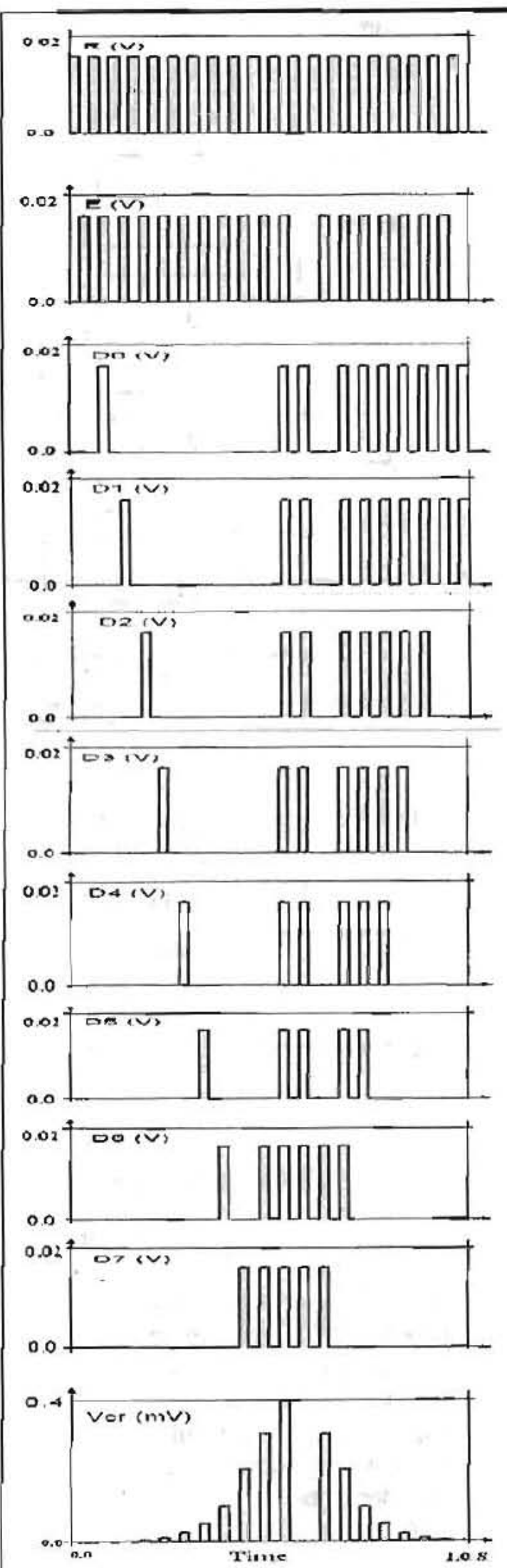

Fig. 3 Simulation results for the 8-bit DAC.
Table 4: The values of $V_{c r}$ for the 8-bit DAC SEC

\begin{tabular}{|c|c|c|c|}
\hline Sample & $\begin{array}{l}\text { 8-bit } \\
\text { Input }\end{array}$ & $\begin{array}{c}\text { Enable } \\
\text { (E) }\end{array}$ & $\mathrm{V}_{\mathrm{et}}(\mu \mathrm{V})$ \\
\hline 1 & 0 & “1" & 0.017 \\
\hline 2 & 1 & "1" & 1.619 \\
\hline 3 & 2 & "1" & 3.221 \\
\hline 4 & 4 & "1" & 6.425 \\
\hline 5 & 8 & "l" & 12.834 \\
\hline 6 & 16 & "l" & 25.651 \\
\hline 7 & 32 & "I" & 51.285 \\
\hline 8 & 64 & "l" & 102.553 \\
\hline 9 & 128 & "I" & 203.486 \\
\hline 10 & 192 & "1" & 304.420 \\
\hline 11 & 255 & "1" & 397.343 \\
\hline 12 & 255 & “0” & 0.00358 \\
\hline 13 & 192 & “I" & 304.420 \\
\hline 14 & 127 & "l" & 203.486 \\
\hline .15 & 63 & "l" & 100.951 \\
\hline 16 & 31 & "l" & 49.683 \\
\hline 17 & 15 & "1" & 24.049 \\
\hline 18 & 7 & "I" & 11.232 \\
\hline 19 & 3 & “l" & 4.823 \\
\hline 20 & 3 & “0” & 0.00004 \\
\hline
\end{tabular}

The proposed 8-bit DAC SEC consists of 57 circuit elements; 16 tunnel junctions, 40 capacitors, in addition to 
the charge reservoir capacitor. The proposed 8-bit DAC SEC was implemented and simulated using the SIMON 2 simulator. We discussed the trade off when changing the charge reservoir capacitance value $\mathrm{C}_{c r}$ on the corresponding voltage $\mathrm{V}_{\mathrm{fr}}$.

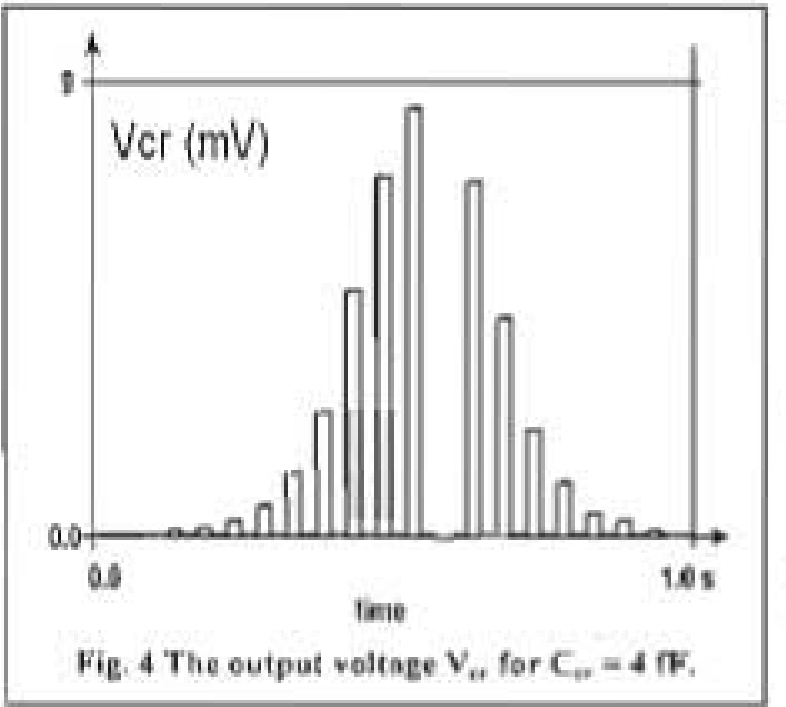

References:

[I] "Techuology Roadmap for Nanoelectronics, "htlp://www cordis.lu/esprit/ src/melna.hum, 1999, published online by the Microelectronics Advanced Research Initiative - MELARI NANO.

[2] K. Likharev, "Single-Electron Devices and Their Applications," Proc. IEEE, vol. 87, no. 4, pp. 606-632, Apr. 1999.

[3] S. Cotofana, C. Lageweg, and S. Vassilidis, "Addition Related Arithmetic Operations via Controlled Transport of Charge," IEEE Trans. On Computers, vol. 54, no. 3, pp. 243-256, Mar. 2005.
[4] A.N. Korotkov, "Coulomb Blockade and Digital Single-Electron Devices," Molecular Electronics, edited by $J$. Joriner el. al, Blackwell, Oxford, 1997. [5] D.G. Gorden, et. al., "Overview of nanoelectronic devices," Proc. IEEE, vol. 85, no. 4, pp. 521-540, 1997.

[6] C. Lageweg. S. Cotofana, and S. Vassilladis, "A Linear Threshoid Gate Implementation in Single Electron Technology," in IEEE Computer Society Workshop on VLSI, pp. 93-98, April 2001.

[7] C. Lageweg. S, Cotofana, and S. Vassilladis, "Single Electron Encoded Latches and Flip-Flops," IEEE Trans. On Nanotechnology 2, vol. 3, pp. 237. 248. June 2004.

[8] S.-W. Jung. B.-H. Lee, and $\mathrm{Y},-\mathrm{H}$. Jeong. "Digital Quantizer based on Single Electron Box for Multi-valued Logic Circuits," Proceedings of the $5^{\text {th }}$ IEEE Conference On Nanotechnology. pp. (CD proceedings), Nagoya, Japan, July 2005.

[9] R. van de Haar and J. Hoekstra, "Simulation of a Neural Node Using SET Technology," A.M. Tyrrel, et. al. (Eds.), Springer-Verlag Berlin Heidelberg. ICES 2003, pp. 377-386, 2003.

[10] S. Rehan, "A Novel Half-Adder Using Single Electron Tunneling (SET) Technology," The $2^{\text {nd }}$ Annual IEEE International Conference on NEMS, Bangkok, Thailand, Jan. 16-19, 2007.

[II] C. Wasshuber, SIMON 2.0 (SIMulation or Nanostructures) hup//www. Ivbrary.com/simon/ 